\title{
Traffic Modelling For Capacity Analysis of CDMA Networks Using Lognormal Approximation Method
}

\author{
J.J. Biebuma and B.O. Omijeh \\ ${ }^{1,2,}$ Department of Electrical/Electronic Engineering University of Port Harcourt Nigeria
}

\begin{abstract}
This paper presents teletraffic modelling in cellular networks operating with Code Division Multiple Access (CDMA) to assist in capacity analysis of such systems using statistical means. Since the introduction of CDMA, owing to capacity edge over other multiple access techniques, there is the need for tools that will assist in ensuring quality of service and proper network dimensioning. This work produces a model useful for capacity analysis. This is achieved by modelling telephone traffic using lognormal assumptions to generate a CDMA blocking probability that is adapted into Erlang $B$ formula for capacity calculations. MATLAB is used to realise the blocking probability formula so developed such that numerical and graphical results are outputted. Results show the effect of variations in network parameters on CDMA. Thus the model is applicable in network planning and dimensioning.

Keywords: Teletraffic, Cellular network, CDMA, Erlang, Modelling, Lognormal
\end{abstract}

\section{Introduction}

Code Division Multiple Access (CDMA) is the preferred access technique for mobile communications mainly for its capacity reasons [1]. Its advantage over other multiple access schemes (like FDMA and TDMA) include higher spectral reuse efficiency, greater immunity to multipath fading, more robust handoff procedures, gradual overload capability and voice activity effects.

In CDMA however, the separation between traffic and transmission issues is not clear with capacity being determined by interference caused by all the transmitters in the network [2]. This calls for more researches to understand its traffic behaviour

Cooper in his work (3) carried out analysis of cell interference in spread spectrum. An extension of this which includes the effects of shadowing and voice activity monitoring, is found in the work of Gilhousen in 1991. In the paper [4] by Fapojuwo in 1993, a computationally intensive procedure is presented for the evaluation of the teletraffic capacity of both forward and reverse links in a CDMA cellular system. Kim in 1993, did a very similar analysis with the exception that the fixing of the PLE at 4 leads to analytic expressions for the interference from the circular cells[5]. This is extended to an analytic result for variance [6]. A standard hexagonal cellular layout is assumed with the propagation model in the paper [7] by Kohno et al in 1995, which includes lognormal shadowing taken to be independent on distinct paths.An extension of the reverse link analysis of the work by Gilhousen et al is discussed in a paper by Viterbi et al [8]. Robert, in 1996 carried out an investigation of the effect of using the actual distance of users when calculating the capacity of a CDMA network [9][10]. Simulations were carried out for twenty-seven cell CDMA network. The simulation results show that for a uniform user distribution, the difference in capacity determined using relative actual interference and relative average interference is too small to warrant the incursion of heavy computational load involved in the former case. This discovered advantage is explored in the capacity analysis in this paper .Blocking of subscribers trying to make calls occurs when the reverse link multiple access interference power reaches a predetermined level that is set to maintain acceptable signal quality .

\section{Traffic Modelling Of Mobile Cellular Systems}

The traffic offered to a network is a measure of the number of call initiations and the duration of calls. It is defined as a product of the mean arrival rate (calls/sec) and the mean call holding time $(\mathrm{sec})$. The dimensionless unit of traffic is the Erlang.

\section{A. Traffic Load and Trunk Size}

The amount of traffic being "offered" to the switch that routes calls over the links is called the traffic load and has the measure Load $=$ Call rate $\mathrm{x}$ Call duration

$$
\mathrm{M}=\mathrm{A} / \mathrm{A}_{0}
$$




\section{B. Erlang B Statistics}

The arrival of calls from different users at the switch under these conditions is assumed to be a Poisson

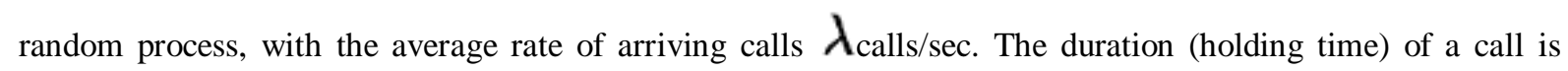
assumed to have an exponential probability distribution, with an average call length of $\mathrm{T}$ seconds. This distribution is valid for a call departure or completion process that is Poisson, with average departure rate of $1 / \mathrm{T}$ $=\mu$ calls/sec. As the random call traffic arrives and departs, the number of lines occupied by ongoing calls can vary from 0 to $\mathrm{N}$. The number of lines occupied is restricted to $0<\mathrm{k}<\mathrm{N}$. Where $\mathrm{k}$ is the active call per time. The state of having all lines occupied may be momentary, so it is referred to as "time congestion." If calls are rejected when all $N$ lines are occupied, then $P_{k}$ for the case of $\mathrm{k}=\mathrm{N}$ is the probability that a call is rejected or "blocked":

$$
B=P_{N}=\frac{(\lambda / \mu)^{N} / N !}{\sum_{k=0}^{N}(\lambda / \mu)^{k} / k !}=\frac{A^{N} / N !}{\sum_{k=0}^{N} A^{k} / k !}, A=\frac{\lambda}{\mu}
$$

This expression for the blocking probability is known as the Erlang B formula. The blocking probability is also a function of the dimensionless quantity.

$$
\frac{\lambda(\text { call } / \text { sec })}{\mu(\text { calls } / \text { sec })}=\mathrm{A}(\text { traffic "load" in Erlangs })
$$

For a finite number of users $(\mathrm{M})$, the load in Erlangs can also be expressed in Terms of $\rho$, the fraction of time that each user occupies a telephone line. Thus

$$
A=M \rho \text {. }
$$

The offered load $A$ can be interpreted as the average number of lines occupied when the blocking probability is small.

\section{CDMA Blocking Probability Modeling}

The determination of Erlang capacity depends on the assumptions about the probability distributions of the call traffic and user interference. In this paper, CDMA Erlang capacities are determined using lognormal approximations.

\section{A. Formulation of the Blocking Probability}

First we consider a single, isolated CDMA cell with M active users. The total reverse link signal-plusnoise power received at the base station can be written as

$$
\underbrace{\alpha_{r 1} P_{1}+\alpha_{r 2} P_{2}+\ldots \ldots+\alpha_{r M} P_{M}+\left(N_{0} W\right)_{c}}_{\text {M reverse link signals }}
$$

Where

- The $\left\{\alpha_{r i}\right\}$ are random variables representing the reverse link voice activity, which have the experimental values given as

$\mathrm{E}\left\{\alpha_{r i}\right\}=\overline{\alpha_{r}}=0.4$ and $\mathrm{E}\left\{\alpha_{r i}^{2}\right\}=\overline{\alpha_{r}^{2}}=0.31$

- The $\left\{P_{i}\right\}$ are the random signal powers for the M active users.

- The number of signals $\mathrm{M}$ is itself an RV, assumed to have a Poisson distribution, so that $\mathrm{E}\{M\}=\bar{M}=\operatorname{Var}\{M\}$

To a potential $(M+1)$ st reverse link user, the total power for the $M$ active users and the thermal noise is interference power. Thus,

Where is the power spectral density level for the total received interference power.

Normalized by $I_{0}^{\prime} R_{b}$, where $R_{b}$ is the data bit rate, the total interference is characterized by the quantity 


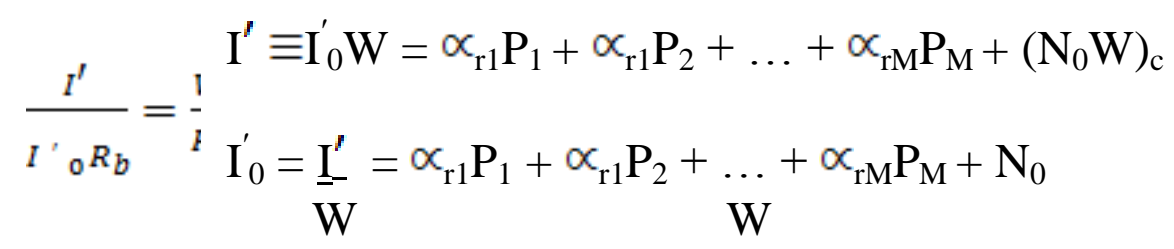

$\frac{I^{I}}{I^{{ }^{r}{ }_{0} R_{b}}}=\frac{W}{R_{b}}=\propto_{r 1} \frac{E_{b 1}}{I_{0}^{I}}+\propto_{r 2} \frac{E_{b 2}}{I_{0}^{r}}+\cdots+\propto_{r M} \frac{E_{b M}}{I_{0}^{I}}+\frac{N_{0}}{I_{0}^{I}} \times \frac{W}{R_{b}}$

Where $\mathrm{P}=\mathrm{E}_{b} \mathrm{R}_{\mathrm{b}}$

$$
\begin{gathered}
\triangleq \sum_{i=1}^{M} \propto_{r 1} \rho_{i}=\frac{W}{R_{b}}(1-\eta) \\
\rho_{i} \triangleq E_{b i} / I_{0}^{F} \\
\eta \triangleq \frac{N_{0}}{I_{0}^{s}} \quad \text { (thermal noise) }
\end{gathered}
$$

Where $\mathrm{P}=\mathrm{E}_{b} \mathrm{R}_{\mathrm{b}}$ is a parameter indicating the loading of the CDMA system and $W / \mathrm{R}_{\mathrm{b}}$ is the spread-spectrum processing gain. Given the value of $\eta$, the quality of the channel that is available to the $(M+1)$ st mobile user is characterized by the value of the random variable $Z$; if $Z$ exceeds some threshold value, then the channel is effectively unavailable (blocked) to the $(M+1)$ st user. In terms of the distribution of the random variable $Z$, the probability that the $(M+1)$ st mobile CDMA user will be blocked is the probability that $Z$ exceeds some threshold value $Z_{0}$, as a function of a threshold value of the interference parameter $\eta_{0}$.

$$
\begin{aligned}
\mathrm{B}_{\mathrm{CDMA}} & =\operatorname{Pr}\left\{\mathrm{Z}>\mathrm{Z}_{0}=\frac{W}{R_{b}}\left(1-\eta_{0}\right)\right\} \\
& =\operatorname{Pr}\left\{\sum_{i=1}^{M} \alpha_{r i} \rho_{i}>\frac{W}{R b}\left(1-\eta_{0}\right)\right\}
\end{aligned}
$$

If a probability density function $\mathrm{p}_{\mathrm{z}}(\mathrm{x})$ is known or assumed for $\mathrm{Z}$, then the evaluation of $\mathrm{B}_{\mathrm{CDMA}}$ is simply a matter of integrating that pdf over the region defined by $Z>Z_{0}$ :

$$
\mathrm{B}_{\mathrm{CDMA}}=\int_{Z_{0}}^{\infty} d x p_{z}(x)
$$

The exact $p d f$ of $Z$ is not known, however, so an approximation is needed to compute $\mathrm{B}_{\mathrm{CDMA}}$.

The CDMA blocking probability can be manipulated to the form

$$
\begin{aligned}
& \mathrm{B}_{\mathrm{CDMA}}=\operatorname{Pr}\left\{Z>Z_{0}\right\}=\operatorname{Pr}\left\{\begin{array}{c}
Z-E\{Z\} \\
\sqrt{\operatorname{Var}\{Z\}}>\frac{Z_{0}-E\{Z\}}{\sqrt{\operatorname{Var}\{Z\}}}
\end{array}\right\} \\
& =\mathrm{Qz}\left(\begin{array}{c}
Z_{0}-E\{Z\} \\
\sqrt{\operatorname{Var}\{Z\}}
\end{array}\right)
\end{aligned}
$$

Where $\mathrm{Q} z(\cdot)$ is notation for the complementary cumulative distribution function of the standardized (zero-mean, unit variance) version of the RV Z. 
The approximation method to be considered in what follows is Lognormal approximation: Based on the fact the SNRs in the sum are lognormal, thus, $Z$ itself can be approximately characterized as a lognormal variable. The approximation method is based on identifying the actual mean and variance of $Z$ with the mean and variance of a lognormal RV. Next, we find the mean and variance of $Z$ and specify its relations to lognormal RV.

\section{B. $\quad$ Mean and Variance of $Z$}

The form of the interference statistics $Z$ is the weighted sum of the $M R V s$

$\{\rho \mathrm{i}, \mathrm{i}=1,2, \ldots, M\}$

$$
\begin{aligned}
& Z=\propto_{r 1} \frac{E_{b 1}}{I_{0}^{I}}+\propto_{r 2} \frac{E_{b 2}}{I_{0}^{I}}+\ldots+\alpha_{r M} \frac{E_{b M}}{I_{0}^{I}} \\
& =\propto_{r 1} \rho_{1}+\propto_{r 2} \rho_{2}+\cdots+\propto_{r M} \rho_{M}
\end{aligned}
$$

The $\{\rho \mathrm{i}\}$ above, when measured in $\mathrm{dB}$ units, are close to having a Gaussian probability distribution with median $\mathrm{m}_{d B}$ and standard deviation $\sigma_{\mathrm{dB}}$ :

$$
\rho_{i}(\mathrm{~dB})=10 \log _{10} \rho \mathrm{i}=m_{\mathrm{dB}}+\sigma_{\mathrm{dB}} \mathrm{G}_{\mathrm{i}}, \quad \mathrm{G}_{\mathrm{i}}=\mathrm{G}(0,1)
$$

Therefore, the RV $\rho_{\mathrm{i}}$ is lognormal and can be written

$$
\begin{aligned}
& \rho_{\mathrm{i}}=10^{\left(\mathrm{m}_{\mathrm{dB}}+\rho_{\mathrm{dB}} \mathrm{Gi}\right) / 10}=\left(\mathrm{e}^{\ln 10}\right)^{\left(\mathrm{m}_{\mathrm{dB}}+\rho_{\mathrm{dB}} \mathrm{Gi}\right) / 10} \\
& =\mathrm{e}^{\beta(\mathrm{mdB}+\sigma \mathrm{dBGi})}, \quad \text { using } \beta=(\operatorname{In} 10) / 10
\end{aligned}
$$

The median, mean, and mean square of $\rho_{\mathrm{i}}$ are assumed to be same for all $\mathrm{i}$ and are obtained as follows:

- Median

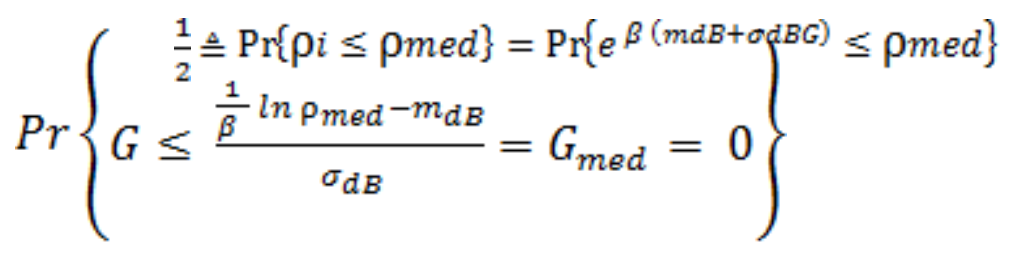

Thus $\quad \rho_{\text {med }=e^{\beta m_{\mathrm{dB}}}}$

- Mean

$$
\begin{aligned}
& \mathrm{E}\{\rho \mathrm{i}\}=\mathrm{E}\left\{e^{\beta(m d B+\sigma d B G)}\right\}=e^{\beta m d B} E\left\{e^{\beta \sigma d B G}\right\} \\
& =\text { omed } \mathrm{E}\left\{e^{u G}\right\} \mid u=\beta \sigma d B
\end{aligned}
$$

Where

$$
\mathrm{E}\left\{e^{u G}\right\}=M_{G}(\mathrm{u})=e^{u^{2} / 2} \quad(M G F)
$$

Thus

$$
\mathrm{E}\{\rho \mathrm{i}\}=\rho_{\mathrm{med}} \mathrm{M}_{\mathrm{G}}\left(\beta \sigma_{d B}\right)=\rho_{\text {med }} e^{\frac{(\beta \sigma d B)^{2}}{2}}
$$

Means square 
The mean, mean square and variance of $Z$ therefore are

$$
\begin{aligned}
& E\{Z\}=E_{M}\{E\{Z \mid M\}\}=E_{M}\left\{\sum_{i=1}^{m} E\left\{\alpha_{r i} \rho_{i}\right\}\right\} \\
& =E_{M}\left\{M E\left\{\alpha_{r i} \rho_{i}\right\}\right\}=E\{M\} E\left\{\alpha_{r i} \rho_{i}\right\} \\
& =\bar{M}_{\text {I=j term }} \bar{M} \bar{\alpha}_{\text {, }} e^{\beta d m B+\frac{1}{2} \beta^{2} \sigma^{2} d B} \\
& \text { Ifj terms } \\
& E\left\{Z^{2}\right\}=E_{M}\left\{E\left\{Z^{2} \mid M\right\}\right\}=E_{M}\left\{\sum_{i=1}^{M} \sum_{j=1}^{M} E\left\{\alpha_{r i} \alpha_{r j} \rho_{i} \rho_{j}\right\}=E_{M}\right. \\
& \left\{M E\left\{\alpha_{r i}^{2} \rho_{i}^{2}\right\}+M(M-1)\left[E\left\{\alpha_{r i} \rho_{i}\right\}\right]^{2}\right\} \\
& \left.=E_{M}\left\{M\left\{E\left\{\alpha_{r i}^{2} \rho_{i}^{2}\right\}+\left[E\left\{\alpha_{r i} \rho_{i}\right\}\right]^{2}\right\} \overline{M^{2}} E\left\{\alpha_{r i} \rho_{i}\right\}\right]^{2}\right\} \\
& =\bar{M} \operatorname{Var}\left\{\alpha_{r i} \rho_{i}\right\}+\overline{M^{2}}\left[E\left\{\alpha_{r i} \rho_{i}\right\}\right]^{2} \\
& \operatorname{Var}\{Z\}=E\left\{Z^{2}\right\}-[E\{Z\}]^{2} \\
& =\bar{M} \operatorname{Var}\left\{\alpha_{r i} \rho_{i}\right\}+\left(\overline{M^{2}}-\bar{M}^{2}\right)\left[E\left\{\alpha_{r i} \rho_{i}\right\}\right]^{2} \\
& =\bar{M} \operatorname{Var}\left\{\alpha_{r i} \rho_{i}\right\}+\operatorname{Var}\{M\}\left[E\left\{\alpha_{r i} \rho_{i}\right\}\right]^{2} \\
& \left.=\bar{M} \operatorname{Var}\left\{\alpha_{r i} \rho_{i}\right\}+\left[E\left\{\alpha_{r i} \rho_{i}\right\}\right]^{2}\right\}, \quad \text { because } \operatorname{Var}\{M\}=\bar{M} \\
& =\bar{M} E\left\{\alpha_{r i}^{2} \rho_{i}^{2}\right\}=\bar{M} \overline{\alpha_{r}^{2}} e^{2 B m d B+2 \beta^{2} \sigma_{d B}^{2}}
\end{aligned}
$$

The interference due to mobiles in other cells can be accounted for by using first- and second-order frequency reuse factors $F=1+\xi$ and $\mathrm{F}^{1}=1+\xi^{\prime}$, respectively, where

$$
\xi=\frac{\text { Total other cell received (median)power }}{\text { Total same }- \text { cell received (median)power }}
$$

and

$$
\xi^{\prime}=\frac{\text { Total other cell mean square received power }}{\text { Total other cell mean square received power }}
$$

A typical analytical value of $\xi^{\prime}=0.086$ and $\xi=\xi^{\prime}=0.55$. With this method of accounting for interference from other cells, the mean and variance for $Z$ become

And

$$
\begin{aligned}
& \mathrm{E}\{\mathrm{Z}\}==\bar{M} \overline{\alpha r} \rho_{\text {med }} e^{\left(\frac{1}{2}\right) \beta^{2} \sigma_{d B}^{2}} \cdot(1+\xi) \\
& \operatorname{Var}\{\mathrm{Z}\}=\bar{M} \overline{\alpha_{r}^{2}} \rho_{\text {med }}^{2} e^{2 \beta^{2} \sigma_{d B}^{2}} \cdot\left(1+\xi^{\prime}\right)
\end{aligned}
$$

\section{Approximations for The Probability Distribution of $Z$}


Because the $M$ RVs $\left\{\rho_{i}, \mathrm{i}=1,2 \ldots \mathrm{M}\right\}$ are lognormal $\mathrm{RV}$, the interference statistic $\mathrm{Z}$ is the weighted sum of lognormal RVs. One approximation for the distribution of $Z i$ s based on assuming that the summing of variables to produce $Z$ causes its distribution to converge to a Gaussian distribution according to the CLT. Another approach is to assume that the lognormal character of the $\left\{\rho_{i}\right\}$ makes $Z$ have an approximately lognormal distribution.

Thus:

$$
\begin{gathered}
\mathrm{Z}=\propto_{r 1} \rho_{1}+\propto_{r 2} \rho_{2}+\cdots+\propto_{r M} \rho_{M} \\
=\propto_{r 1} e^{\beta\left(m d B+\sigma d B G_{1}\right)}+\propto_{r 2} e^{\beta\left(m d B+\sigma d B G_{2}\right)+\cdots}+\propto_{r M} e^{\beta\left(m d B+\sigma d B G_{M}\right)} \\
\approx e^{m_{M}+\sigma_{M} G} \quad \text { Lognormal approximation }
\end{gathered}
$$

CDMA Blocking Probability Formula for Lognormal Assumptions

Under lognormal assumption, the mean and variance of $Z$ are identified as the mean and variance of lognormal $\mathrm{RV}, \zeta$, where

$$
\zeta=e^{m_{M+\sigma} m_{G}}
$$

The mean, mean square, and variance of $\zeta$ are given by

$$
\begin{aligned}
& \mathrm{E}\{\zeta\}=e^{m_{M}} E\left\{e^{\sigma_{M} G}\right\}=e^{m_{M}+\frac{1}{2} \sigma^{2}{ }_{M}} \\
& \mathrm{E}\left\{\zeta^{2}\right\}=e^{2 m_{M}} E\left\{e^{2 \sigma_{M} G}\right\}=e^{2 m_{M}+2 \sigma_{M}^{2}} \\
& \operatorname{Var}\{\zeta\}=\mathrm{E}\left\{\zeta^{2}\right\}-[\mathrm{E}\{\zeta\}]^{2}=e^{2 m_{M}+\sigma_{M}^{2}}\left[e^{\sigma_{M}^{2}}-1\right]
\end{aligned}
$$

By setting $\mathrm{E}\{Z\}=\mathrm{E}\{\zeta\}$ and $\operatorname{Var}\{Z\}=\operatorname{Var}\{\zeta\}$, where the mean and variance of $Z$ are already given. solving for $\mathrm{m}_{\mathrm{M}}$ and $\sigma_{\mathrm{M}}^{2}$ :

$$
\begin{gathered}
\bar{M} \overline{\alpha_{r}} e^{\beta m_{d B}+\frac{1}{2} \beta^{2} \sigma_{d B}^{2}(1+\xi)=e^{m_{M}+\frac{1}{2} \sigma_{M}^{2}}} \text { and } \\
\bar{M} \overline{\alpha_{r}^{2}} e^{2 \beta m_{b B}+2 \beta^{2} \sigma_{d B}^{2}}\left(1+\xi^{1}\right)=e^{2 m_{M}+\sigma_{M}^{2}}\left[e^{\sigma^{2} m}-1\right]
\end{gathered}
$$

The solution is

$$
\begin{aligned}
& \sigma_{M}^{2}=\ln \left[\frac{\overline{\alpha_{r}^{2}}\left(1+\xi^{\prime}\right) e^{\beta^{2} a_{d B}^{2}}}{\bar{M}\left(\alpha_{r}\right)^{2}(1+\xi)^{2}}+1\right] \\
& m_{M}=\operatorname{In}\left[\bar{M} \overline{\alpha_{r}}(1+\xi)\right]+\beta m_{d B}+1 / 2\left(\beta^{2} \sigma_{d B}^{2}-\sigma_{M}^{2}\right)
\end{aligned}
$$

Using these parameters, the blocking probability formula for the lognormal approximation is

$$
\mathrm{B}_{\mathrm{CDMA}}=\operatorname{Pr}\left\{\mathrm{Z}>\mathrm{Z}_{\mathrm{O}}\right\} \approx \operatorname{Pr}\left\{e^{m+\sigma_{M} G}>\mathrm{Z}_{0}\right\}_{=} \mathrm{Q}\left(\frac{\ln Z_{0-m_{M}}}{\sigma_{M}}\right)
$$

Substituting the expressions for $\mathrm{m}_{\mathrm{M}}$ and $\sigma_{\mathrm{M}}$, we obtain general expressions for the CDMA blocking probability under the lognormal approximation for the interference statistic, given by

$\mathrm{B}_{\mathrm{CDMA}}=\mathrm{Q}\left(\frac{\ln \left[\frac{W}{R_{b}}\left(1-\mathrm{\eta}_{0}\right)\right]-\ln \left[\bar{M} \overline{\alpha_{r}}(1+\xi)\right]-\beta m_{d B}}{\sqrt{\ln \left[\frac{\overline{\alpha_{r}^{2}}\left(1+\xi^{\prime}\right) e^{\beta^{2} \sigma_{d B}^{2}}}{\overline{M(\alpha)^{2}}(1+\xi)^{2}}+1\right]}}\right.$ 


$$
\left.-\frac{\left.\frac{1}{2}\left\{\beta^{2} \sigma_{d B}^{2}-\ln \left[\frac{\overline{\alpha_{T}^{2}}\left(1+\xi^{\prime}\right) e^{\beta^{2} \sigma_{d B}^{2}}}{\overline{M(\bar{\alpha})^{2}}(1+\xi)^{2}}+1\right]\right\}\right)}{\sqrt{\ln \left[\frac{\overline{\alpha_{T}^{2}}\left(1+\xi^{\prime}\right) e^{\beta^{2} \sigma_{d B}^{2}}}{\overline{M(\alpha)^{2}}(1+\xi)^{2}}+1\right]}}\right)
$$

in which the Erlang capacity is $\bar{M}$. Because the interference parameter $\eta$ is $\eta=1-\mathrm{X}$, we may convert the threshold $\eta_{0}$ into a loading threshold $X_{0}=1-\eta_{0}$ and write (3.72) as

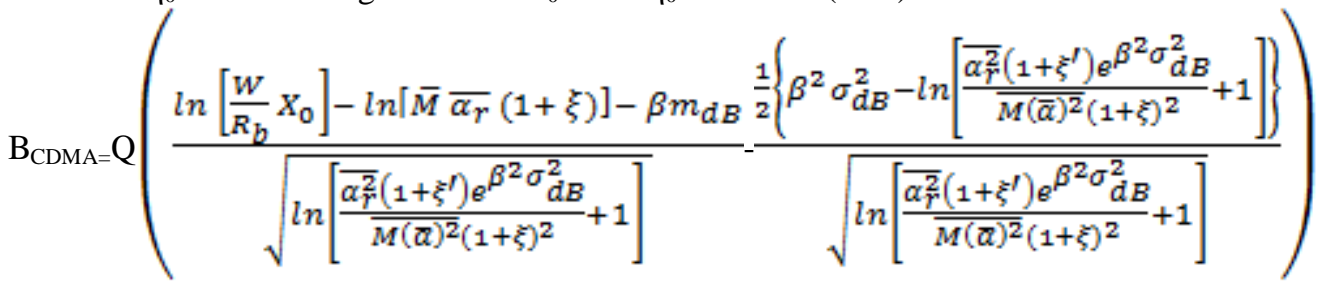

\section{A. Graphical Plots and Analysis of CDMA Blocking Probability Formula for Lognormal Assumptions}

The blocking probability formula derived with Lognormal Assumptions in the previous section is as follows:

$$
\mathrm{B}_{\mathrm{CDMA}}=\mathrm{Q}\left(\frac{\ln \left[\frac{W}{R_{b}} X_{0}\right]-\ln \left[\bar{M} \overline{\alpha_{r}}(1+\xi)\right]-\beta m_{d B}}{\sqrt{\ln \left[\frac{\alpha_{r}^{2}\left(1+\xi^{\prime}\right) e^{\beta^{2} \sigma_{d B}^{2}}}{\overline{M(\alpha)^{2}}(1+\xi)^{2}}+1\right]}}-\frac{\left.\frac{1}{2}\left\{\beta^{2} \sigma_{d B}^{2}-\ln \left[\frac{\overline{\alpha_{r}^{2}}\left(1+\xi^{\prime}\right) e^{\beta^{2} \sigma_{d B}^{2}}}{\overline{M(\bar{\alpha})^{2}}(1+\xi)^{2}}+1\right]\right\}\right)}{\sqrt{\ln \left[\frac{\alpha_{r}^{2}\left(1+\xi^{\prime}\right) e^{\beta^{2} \sigma_{d B}^{2}}}{\overline{M(\alpha)^{2}}(1+\xi)^{2}}+1\right]}}\right)
$$

Plots of equation (41) for $\mathrm{B}_{\mathrm{CDMA}}$ versus $\bar{M}$ are shown in Figure 1 for a single cell $\left(\xi=\xi^{\prime}=0\right)$ and for multiple cells, using $\xi=\xi^{\prime}=0.55$ and the parameter values $\sigma_{\mathrm{dB}}=2.5 \mathrm{~dB}, W=1.2288 \mathrm{MHz}, \mathrm{R}_{\mathrm{b}}=9.6 \mathrm{kbps}, \mathrm{X}_{0}=0.9, \overline{\alpha_{r}}=$ 0.4 , and $\overline{\alpha_{r}^{2}}=0.31$. The plots are parametric in $\mathrm{m}_{\mathrm{dB}}=\mathrm{E}_{\mathrm{b}} / \mathrm{N}_{0}$, which takes the values 5,6 , and $7 \mathrm{~dB}$.

When $B_{C D M A}=10^{-2}=0.01=1 \%$, figure 1 shows for multiple cells that the corresponding (integer) value of the Erlang capacity $\bar{M}$ is 16 for $\mathrm{E}_{\mathrm{b}} / \mathrm{N}_{0}=7 \mathrm{~dB}, 22$ for $\mathrm{E}_{\mathrm{b}} / \mathrm{N}_{0}=6 \mathrm{~dB}$, and 29 for $\mathrm{E}_{\mathrm{b}} / \mathrm{N}_{0}=5 \mathrm{~dB}$.

$\overline{\alpha_{r}}=0.4, \bar{\alpha}=0.31, X_{0}=0.9, W / R_{b}=128$

In Figure 2, the cell loading threshold $X_{o}$ is varied for the lognormal approximation and the case of $\mathrm{E}_{\mathrm{b}} / \mathrm{N}_{0}=6 \mathrm{~dB}$. Again we see that raising or lowering $\mathrm{X}_{0}$ has a significant effect on the Erlang capacity $\bar{M}$ for a given value of the blocking probability. The amount of increase or decrease in $\bar{M}$ is greater than the amount of increase or decrease in $\mathrm{X}_{0}$. For example, raising $\mathrm{X}_{\mathrm{o}}$ by $20 \%$ from 0.75 to 0.9 increases the value of $\bar{M}$ at $B_{C D M A}=1 \%$ from about 16 to 22 , or about $35 \%$, indicating the high sensitivity of the Erlang capacity to the cell loading. Therefore, the threshold value of cell loading should be chosen very carefully. The sensitivity of the CDMA blocking probability to the value of the second-order reuse fraction $\xi^{\prime}$ under the lognormal approximation is shown in Figure 3, in which the first order reuse fraction $\xi=0.55$ and $\mathrm{E}_{\mathrm{b}} / \mathrm{N}_{0}=5,6$, and $7 \mathrm{~dB}$ are used

$\overline{\alpha_{r}}=0.4, \overline{\alpha_{r}^{2}}=0.31, E_{b} / N_{0}=6 \mathrm{~dB}, W / R_{b}=128$ 


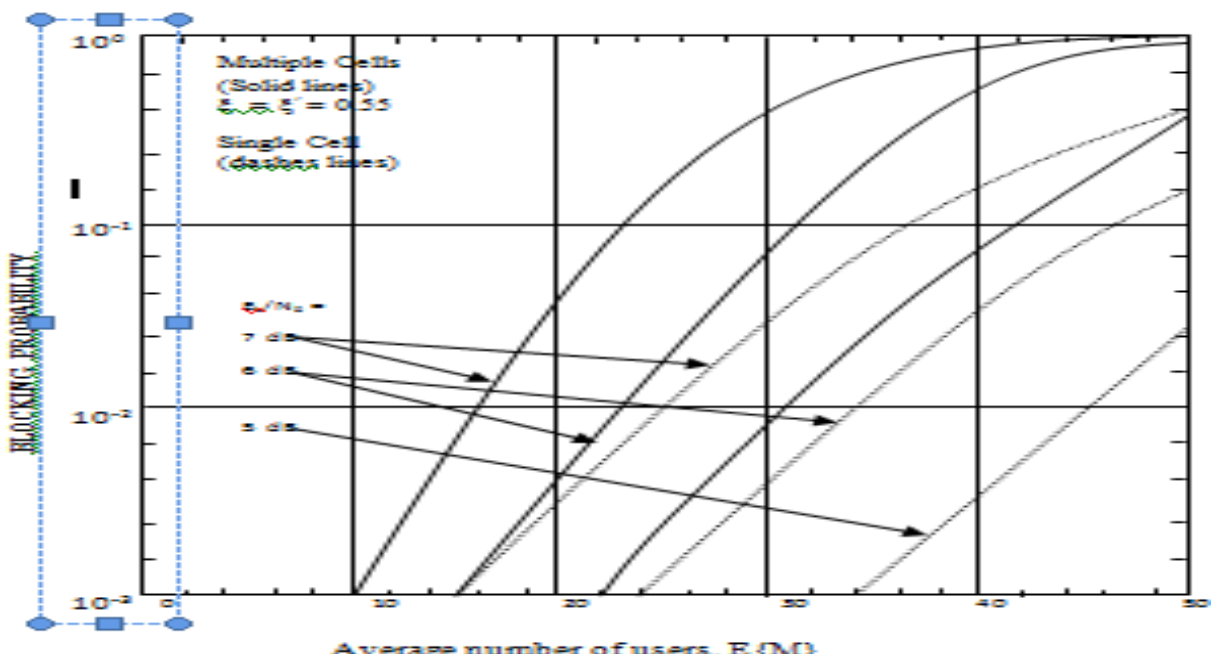

Figure 1: CDMA blocking probability (lognormal approximation) versus average number of mobile users, SNR requirement varied.

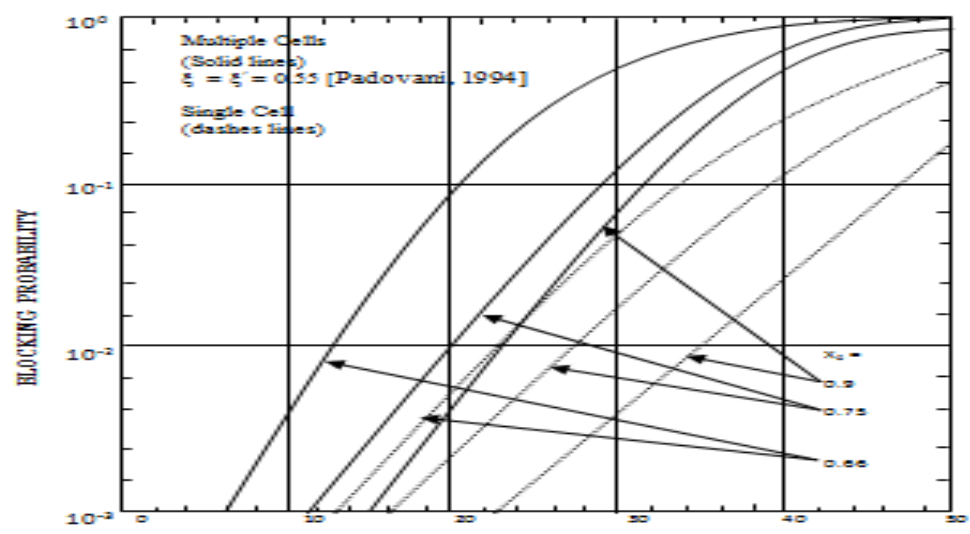

Average number of users, $\mathrm{E}$ M

Figure 2: CDMA blocking probability (lognormal approximation) versus average number of mobile users, loading threshold varied

$$
\overline{\alpha_{r}}=0.4, \overline{\alpha_{r}^{2}}=0.31, X_{0}=0.9 d B, \quad W / R_{b}=128
$$

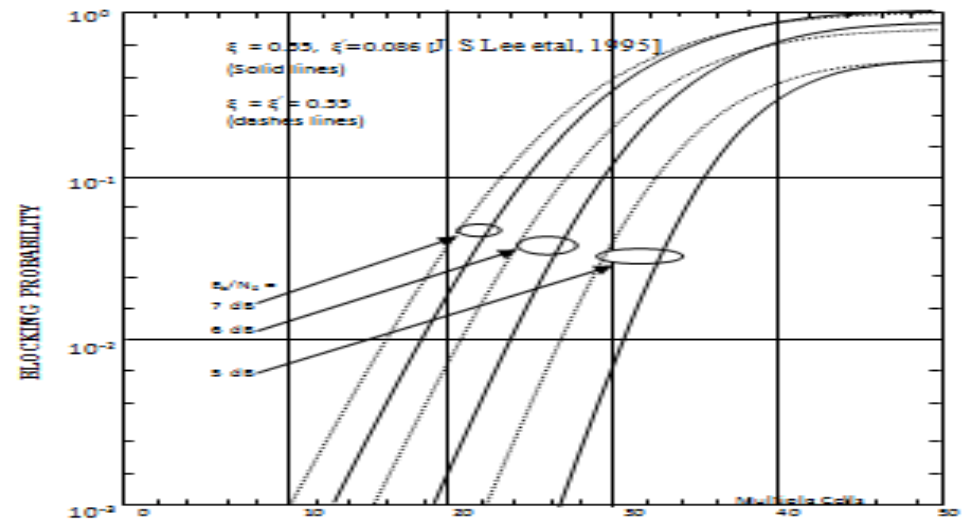

Average number of users, $\mathrm{E}\{\mathrm{M}\}$

Figure 3: CDMA blocking probability (lognormal approximation) versus average number of mobile users, reuse fraction varied 


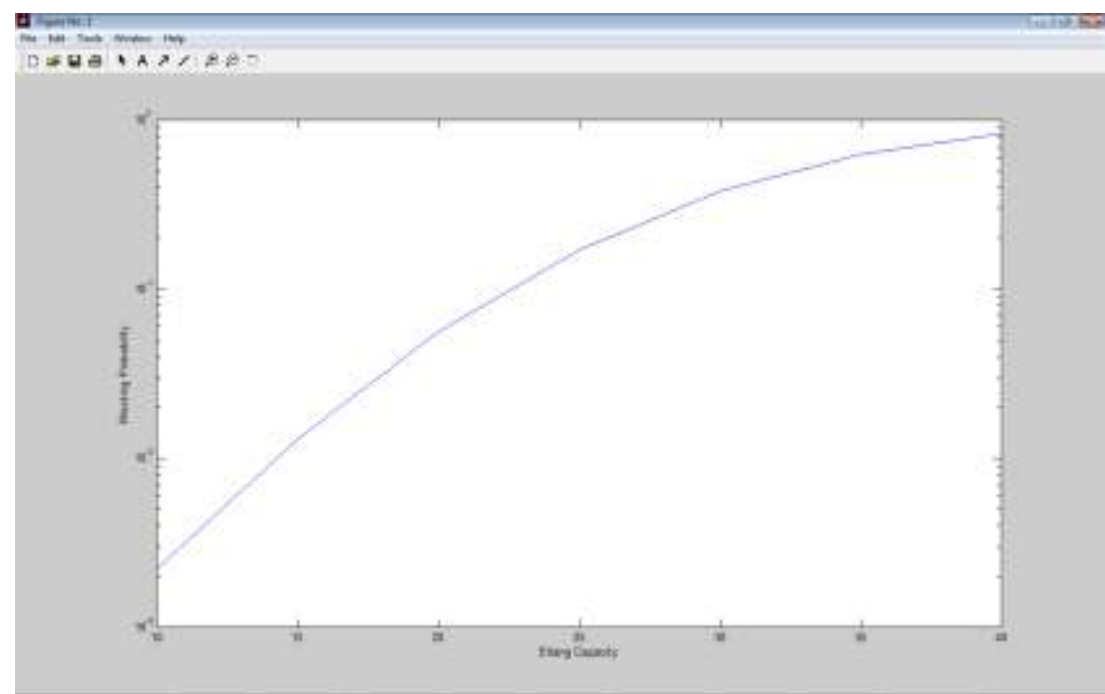

Figure 4: Erlang capacities calculated and results graphically displayed in MATLAB

\section{Conclusion}

We have derived Erlang capacity formulas for the CDMA cellular system under lognormal approximation, on the assumption that the sum of $M$ lognormal Random Variables is also a lognormal RV. The stochastic nature of call arrivals and departures were characterized using statistical means. The interference contributed by each user was modeled as a Poison RV that summed up to statistical RV lognormal characteristics. The blocking probability formula so derived as programmed in MATLAB and Erlang capacities calculated and results graphically displayed. See Figure 4.

Blocking occurred when the reverse link multiple access interference power reached a predetermined level that is set to maintain acceptable signal quality. When the total user interference at a base station receiver exceeded the set threshold, the system blocked the next user attempting to place a call. The number of users for which the CDMA blocking probability equaled $1 \%$ as chosen was taken to be the Erlang capacity of the network. Thus, a new CDMA blocking probability model is developed that enabled the estimation and analysis of Erlang capacity of CDMA networks.

Graphic results for the blocking model generated showed the effect of variations in interference parameters on CDMA capacity. The Erlang capacity from the model is adaptable into Erlang B formula to estimate capacity in terms of channels.

\section{References}

[1] Lee J.S and Miller L.E (1995), "On the Erlang Capacity of CDMA cellular systems," In proc. GLOBECOM'95, Singapore, pp.1877-1883, Nov. 1995.

[2] Evans J.S and Everrit D(1995), "On the teletraffic capacity of CDMA Cellular networks" Department of Computer Engineering and Computer Science, University of California Berkelay, CA, 94720, USA, 1995.

[3] Cooper G.R and Nettleton R.W (1978), “A spread spectrum technique for high capacity mobile communications," IEEE Trans. Veh. Technol., VT-27, pp. 264-275, Nov. 1978.

[4] Fapojuwo A.O (1994), "Radio capacity of Direct sequence code division multiple acess mobile radio systems," in proc. IEEE Vehicular Technology Conference, Stockholm, Sweden, pp. 868-872, June 1994.

[5] Kim K.I(1993)“CDMA cellular engineering issues (1993),” IEEE Trans Veh. Technol. Vol. 42, pp. 345-350,Aug.1993.

[6] Lee J.S and Miller L.E(1998), “CDMA systems Engineering Handbook” Library of Congress Cataloging-in-Publication, Data, 1998.

[7] Kohno R, Meidan R, and Milstein L.B(1995), "Spread spectrum access methods for wireless communications," IEEE Comm. Magazine, vol. 33, pp 58-67, Jan.1995

[8] Viterbi A.J, Viterbi A.M, and Zehavi E(1992), "Soft handoff extends CDMA cell coverage and increases reverse link capacity, “ IEEE J. Select. Areas Commun., vol. 41, pp.231-242, Aug. 1992.

[9] Robert A.K.L and Parvez A (1992), "Impact of interference model on Capacity in CDMA cellular Networks," Department of Computer Science and Engineering, University of North Texas, Denton, TX, 76203.

[10] Jhong S. L. Etal (1993), “CDMA systems Engineering Handbook” Artech House mobile communications Library, 1998. 